\title{
Tricin, 4',5,7-trihydroxy-3',5'-dimethoxyflavone, exhibits potent antiangiogenic activity in vitro
}

\author{
JANG MI HAN $^{1}$, HO JEONG KWON ${ }^{2}$ and HYE JIN JUNG ${ }^{1}$ \\ ${ }^{1}$ Department of BT-Convergent Pharmaceutical Engineering, Sun Moon University, Chungnam 336-708; \\ ${ }^{2}$ Department of Biotechnology, Yonsei University, Seodaemun-gu, Seoul 120-749, Republic of Korea
}

Received April 26, 2016; Accepted July 22, 2016

DOI: $10.3892 /$ ijo.2016.3645

\begin{abstract}
Tumor growth and metastasis depend on angiogenesis triggered by chemical signals, such as vascular endothelial growth factor (VEGF), released from tumor cells. Therefore, the specific perturbation of angiogenesis has been considered a powerful strategy for the treatment of cancer. Herein, we report that tricin, 4',5,7-trihydroxy-3',5'-dimethoxyflavone, exhibits potent antiangiogenic activity in vitro. Tricin effectively suppressed the proliferation as well as VEGF-induced invasion and tube formation of human umbilical vein endothelial cells (HUVECs) at subtoxic doses. Furthermore, tricin significantly inhibited the angiogenesis of the chorioallantoic membrane from growing chick embryos without showing cytotoxicity. We also found that tricin blocked tumor cellinduced angiogenesis. Notably, tricin downregulated not only the VEGFR2 signal transduction by reducing reactive oxygen species (ROS) generation in endothelial cells, but also the expression of VEGF by inhibiting hypoxia inducible factor-1 $\alpha$ (HIF-1 $\alpha$ ) accumulation in tumor cells. Moreover, combined treatment with tricin and bevacizumab, an anti-VEGF drug, ameliorated the antiangiogenic effect of bevacizumab. Taken together, our findings demonstrate for the first time that tricin possesses promising antiangiogenic potential and thus may be applied to anticancer therapy by targeting tumor angiogenesis.
\end{abstract}

\section{Introduction}

Angiogenesis, the formation of new blood vessels from pre-existing vessels, is required for a variety of normal physiological functions such as embryonic development, wound healing and tissue or organ regeneration $(1,2)$. However, angiogenesis is also involved in the pathogenesis of several diseases, including age-related macular degeneration, diabetic

Correspondence to: Professor Hye Jin Jung, Department of BT-Convergent Pharmaceutical Engineering, Sun Moon University, 70 Sunmoon-ro 221, Tangjeong-myeon, Asan-si, Chungnam 336-708, Republic of Korea

E-mail: poka96@sunmoon.ac.kr

Key words: tumor angiogenesis, tricin, VEGFR2, HIF-1 $\alpha$ retinopathy, psoriasis, rheumatoid arthritis and cancer (3). Particularly, upregulation of angiogenesis is a central step in sustained tumor growth and metastasis. The new blood vessels grow and infiltrate into the tumor, providing it with essential nutrients and oxygen, and a route for tumor metastasis $(4,5)$. Therefore, antiangiogenesis has become an important strategy for the treatment of cancer.

Chemical stimulation of angiogenesis is performed by various angiogenic proteins, including vascular endothelial growth factor (VEGF) (6). VEGF stimulates cellular responses by binding to VEGF receptor 2 (VEGFR2) on the cell surface, causing them to dimerize and become activated through transphosphorylation. Activation of VEGFR2 leads to phosphorylation of specific downstream signal transduction mediators, including extracellular signal-regulated kinases (ERK) and AKT. Signaling from VEGFR2 consequently promotes the proliferation, migration and differentiation of endothelial cells $(7,8)$. Therefore, VEGFR2 has been recognized as the most important target for the antiangiogenesis therapy of cancer. Bevacizumab (Avastin ${ }^{\circledR}$ ), sunitinib malate (Sutent $^{\circledR}$, SU11248), and sorafenib (Nexavar ${ }^{\circledR}$, BAY 43-9006) that were developed for antiangiogenic actions have been approved by the United States Food and Drug Administration (FDA) for treatment of patients with specific types of cancer. All three agents inhibit VEGF signaling by blocking VEGF ligand or VEGF receptor function $(9,10)$. But most of angiogenesis inhibitors have some adverse effects, including hypertension and proteinuria, which emphasizes that discovery of novel VEGFR2 inhibitors with better safety and efficacy in treating human cancer is still needed $(11,12)$.

In general, rapid growth of tumor cells causes hypoxia in tumor tissues, which drives angiogenesis to improve the influx of oxygen. Thus, the hypoxic microenvironment can stimulate the expression of VEGF via hypoxia inducible factor-1 (HIF-1), the transcription factor which binds the regulatory region of VEGF gene and induces its transcription during hypoxia $(13,14)$. HIF-1 is a heterodimeric transcription factor composed of an oxygen-regulated $\alpha$-subunit (HIF-1 $\alpha$ ) and a constitutively expressed $\beta$-subunit (HIF-1 $\beta$ ). HIF-1 $\alpha$ plays a key role in the regulation of the expression of many genes involved in metabolic adaptation to low oxygen, survival and angiogenesis. Under normoxic condition, HIF-1 $\alpha$ is rapidly degraded by proteasome after post-translational modification, whereas, under hypoxic condition, it remains stable 


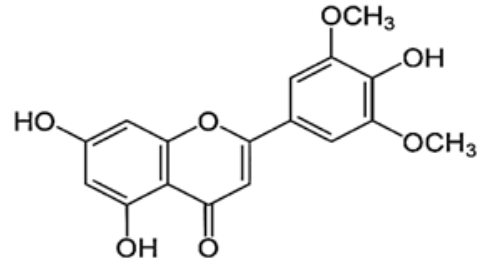

Figure 1. The structure of tricin.

and binds with HIF-1 $\beta$ to activate the transcription of a large number of genes (15). It has been reported that overexpression of HIF-1 $\alpha$ was associated with poor prognosis of many human cancers (16). Given the crucial role of VEGF-mediated signaling in promoting tumor angiogenesis, dual inhibition of VEGFR2 and HIF-1 $\alpha$ activities could potentiates antiangiogenic therapy in cancer treatment.

There has been increasing interest in the research on flavonoids, a large class of plant metabolites, because of their multifaceted health benefits $(17,18)$. The flavonoid, tricin (4',5,7-trihydroxy-3',5'-dimethoxyflavone) was reported as a valuable anticancer agent having a pharmacokinetic advantage (Fig. 1) (19-21). However, the antiangiogenic potential of tricin has not been explored. In the present study, we investigated the in vitro antiangiogenic effect and the molecular mechanisms of tricin. Our results demonstrated that tricin could efficiently suppress tumor angiogenesis by downregulating both VEGFR2 signaling and HIF-1 $\alpha$ activity.

\section{Materials and methods}

Materials. Tricin was purchased from ChemFaces (Wuhan, China). Endothelial growth medium-2 (EGM-2) was obtained from Lonza (Walkersville, MD, USA). Dulbecco's modified Eagle's medium (DMEM), minimum essential medium (MEM), RPMI-1640 medium, and fetal bovine serum (FBS) were purchased from Invitrogen (Grand Island, NY, USA). Recombinant human vascular endothelial growth factor (VEGF), Matrigel and Transwell chamber systems were obtained from Koma Biotech (Seoul, Republic of Korea), BD Biosciences (San Jose, CA, USA) and Corning Costar (Acton, MA, USA), respectively. Anti-hypoxia inducible factor-1 $\alpha$ (HIF-1 $\alpha$ ) antibody was purchased from BD Biosciences. Anti-phospho-VEGFR2, anti-VEGFR2, anti-phosphoAKT, anti-AKT, anti-phospho-ERK1/2, anti-ERK1/2 and anti- $\beta$-actin antibodies were purchased from Cell Signaling Technology (Beverly, MA, USA).

Cell culture and hypoxic conditions. Human umbilical vein endothelial cells (HUVECs) and U87MG (human glioblastoma) cells were grown in EGM-2 and MEM supplemented with $10 \%$ FBS, respectively. AGS (human gastric carcinoma) and HCT116 (human colon carcinoma) cells were maintained in RPMI-1640 medium containing 10\% FBS. HeLa (human cervical carcinoma) and HepG2 (human hepatocarcinoma) cells were grown in DMEM supplemented with $10 \%$ FBS. All cells were maintained at $37^{\circ} \mathrm{C}$ in a humidified $5 \% \mathrm{CO}_{2}$ incubator. For hypoxic conditions, cells were incubated in a hypoxic chamber (Forma Scientific, Marietta, OH, USA) under $5 \% \mathrm{CO}_{2}$ and $1 \% \mathrm{O}_{2}$ balanced with $\mathrm{N}_{2}$.
Cell proliferation assay. HUVECs $\left(3 \times 10^{3}\right.$ cells/well) and various cancer cells $\left(2 \times 10^{3}\right.$ cells/well $)$ were seeded in 96-well culture plates and then treated with various concentrations of tricin for $72 \mathrm{~h}$. Cell proliferation was measured using a 3-(4,5-dimethylthiazol-2-yl)-2,5-diphenyltetrazolium bromide (MTT) colorimetric assay.

Cell viability assay. HUVECs were seeded at a density of $1 \times 10^{5}$ cells/well in 12-well culture plates. Tricin $(1-20 \mu \mathrm{M})$ was added to each well and the cells were incubated for up to $72 \mathrm{~h}$. After $72 \mathrm{~h}$, the cells were stained with Trypan blue and counted using a hemocytometer.

Chemoinvasion assay. The invasiveness of HUVECs was investigated using a Transwell chamber system with polycarbonate filter inserts with a pore size of $8.0 \mu \mathrm{m}$. Briefly, the lower side of the filter was coated with $10 \mu \mathrm{l}$ gelatin $(1 \mathrm{mg} / \mathrm{ml})$ and the upper side was coated with $10 \mu \mathrm{l}$ Matrigel $(3 \mathrm{mg} / \mathrm{ml})$. Serum-starved HUVECs $\left(8 \times 10^{4}\right.$ cells) were placed in the upper chamber of the filter and tricin (2.5-10 $\mu \mathrm{M})$ was added to the lower chamber in the presence of VEGF $(30 \mathrm{ng} / \mathrm{ml})$. The chamber was incubated at $37^{\circ} \mathrm{C}$ for $18 \mathrm{~h}$, and then the cells were fixed with methanol and stained with hematoxylin and eosin (H\&E). The total number of cells that invaded the lower chamber of the filter was counted using an optical microscope (Olympus, Center Valley, PA, USA) at a x100 magnification.

Capillary tube formation assay. Serum-starved HUVECs $\left(8 \times 10^{4}\right.$ cells) were inoculated on a surface containing Matrigel $(10 \mathrm{mg} / \mathrm{ml})$ and were incubated with tricin $(2.5-10 \mu \mathrm{M})$ for $6 \mathrm{~h}$ in the presence of VEGF (30 ng/ml). Morphological changes of the cells and tube formation were visualized under a microscope and photographed at a x100 magnification (Olympus). Tube formation was quantified by counting the total number of branched tubes in randomly selected fields at a x100 magnification.

Chorioallantoic membrane (CAM) assay. Fertilized chick eggs were maintained in a humidified incubator at $37^{\circ} \mathrm{C}$ for 3 days. Approximately $6 \mathrm{ml}$ egg albumin was removed with a hypodermic needle, allowing the CAM and yolk sac to drop away from the shell membrane. After 2 days, the shell was punched out and peeled away. Thermanox coverslips (Nalge Nunc International, Rochester, NY, USA) with or without tricin were air-dried and applied to the CAM surface. Two days later, $2 \mathrm{ml}$ of $10 \%$ fat emulsion (Greencross Co., Yongin, Republic of Korea) were injected into the chorioallantois and the CAM was observed under a microscope.

Tumor cell-induced angiogenesis assay. Tumor-induced angiogenesis was assessed using an in vitro co-culture system based on the chemoinvasion assay (22). U87MG cells were plated in the lower chamber and treated with tricin $(2.5-10 \mu \mathrm{M})$ for $24 \mathrm{~h}$. And then the medium in each lower chamber was replaced with fresh medium without tricin, and serum-starved HUVECs $\left(8 \times 10^{4}\right.$ cells) were placed in the upper chamber. The chamber was incubated at $37^{\circ} \mathrm{C}$ for $18 \mathrm{~h}$, and HUVECs that invaded the lower chamber of the filter were analyzed using the same procedure as described in the chemoinvasion assay. To further verify the activity of tricin to tumor cell-induced 

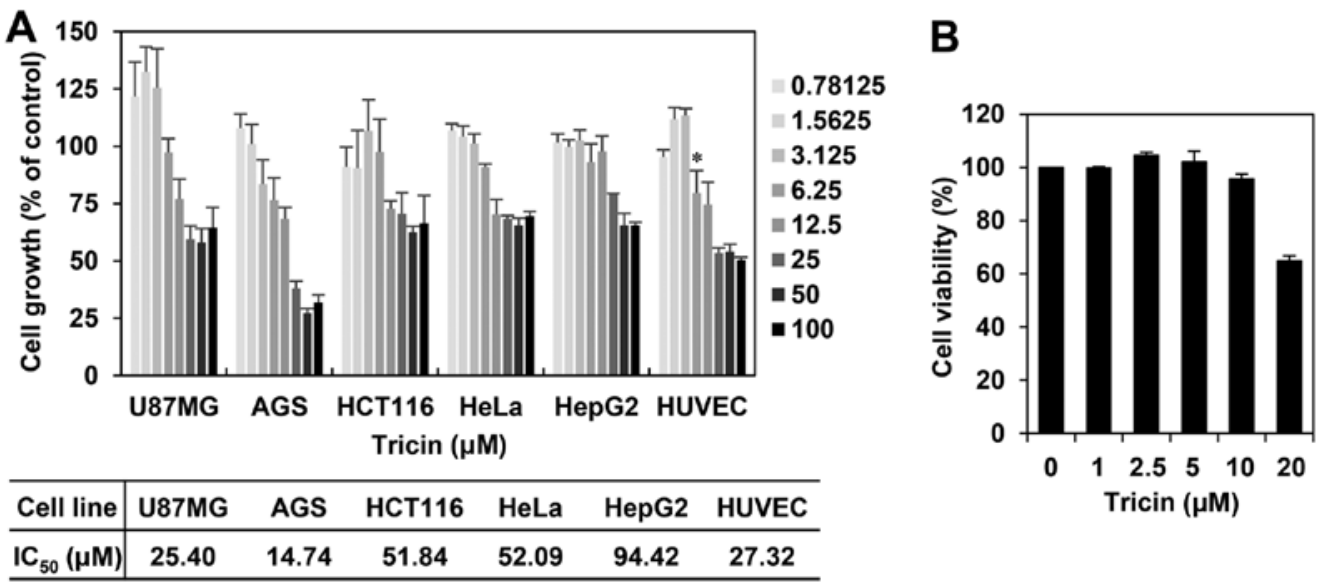

Figure 2. The antiproliferative activity of tricin on HUVECs. (A) The effect of tricin on the growth of HUVECs and a variety of cancer cells. Cells were treated with the various concentrations of tricin and incubated for $72 \mathrm{~h}$. Cell growth was measured using an MTT colorimetric assay. (B) The effect of tricin on the viability of HUVECs. Cells were treated with tricin $(1-20 \mu \mathrm{M})$ and incubated for $72 \mathrm{~h}$. Cell viability was measured by the Trypan blue assay. $\mathrm{P}<0.05$ vs. the control.

angiogenesis, a conditioned medium was collected from U87MG cells and used as the angiogenic stimuli for the tube formation of HUVECs (22). Briefly, U87MG cells were treated with tricin (2.5-10 $\mu \mathrm{M})$ for $24 \mathrm{~h}$, and then the medium was replaced with fresh medium without tricin. The conditioned medium was used in the in vitro tube formation assay.

Western blot analysis. Cell lysates were separated by $10 \%$ sodium dodecyl sulfate-polyacrylamide gel electrophoresis (SDS-PAGE), and the separated proteins were transferred to polyvinylidene difluoride (PVDF) membranes (Millipore, Billerica, MA, USA) using standard electroblotting procedures. The blots were blocked and immunolabeled with primary antibodies against phospho-VEGFR2, VEGFR2, phospho-AKT, AKT, phospho-ERK1/2, ERK1/2, HIF-1 $\alpha$ and $\beta$-actin overnight at $4^{\circ} \mathrm{C}$. Immunolabeling was detected with an enhanced chemiluminescence (ECL) kit (Bio-Rad Laboratories, Hercules, CA, USA), according to the manufacturer's instructions.

Reactive oxygen species (ROS) measurement. ROS levels were detected with 2',7'-dichlorodihydrofluorescein diacetate ( $\mathrm{H}_{2} \mathrm{DCFDA}$; Molecular Probes, Eugene, OR, USA). For the assay, serum-starved HUVECs seeded at a density of $1 \times 10^{5}$ cells/well in 96-black well culture plates were pretreated with tricin (2.5-10 $\mu \mathrm{M})$ for $3 \mathrm{~h}$. After incubation with $\mathrm{H}_{2} \mathrm{DCFDA}$ $(10 \mu \mathrm{M})$ for $5 \mathrm{~min}$, the cells were stimulated to VEGF (30 ng/ml) for $5 \mathrm{~min}$. The fluorescence intensity of DCF was detected using a multimode microplate reader (Thermo Fisher Scientific, Vantaa, Finland) at the excitation and emission wavelengths of 495 and $529 \mathrm{~nm}$, respectively.

Measurement of VEGF by enzyme-linked immunosorbent assay (ELISA). VEGF concentration in the media from the tricin-treated cells was determined using a VEGF immunoassay kit (R\&D Systems, Minneapolis, MN, USA) according to the manufacturer's instructions. The results were expressed as the concentration of VEGF relative to the total amount of protein from each well.
Statistical analysis. The results are expressed as the mean \pm standard error (SE). Student's t-test was used to determine statistical significance between the control and the test groups. A P-value of $<0.05$ was considered to indicate a statistically significant result.

\section{Results}

The effect of tricin on the proliferation of human umbilical vein endothelial cells. We first investigated the effect of tricin on the growth of various cell lines, including several cancer cells and human umbilical vein endothelial cells (HUVECs), using the MTT colorimetric assay. As shown in Fig. 2A, tricin inhibited the proliferation of each cell line with a different sensitivity to growth inhibition. Notably, tricin showed comparatively better inhibition effect on the growth of HUVECs with an $\mathrm{IC}_{50}$ of $27.32 \mu \mathrm{M}$ among the tested cell lines. To further evaluate whether the endothelial cell growth inhibition by tricin was due to cytotoxic or cytostatic activity, a viability assay was performed using the Trypan blue exclusion method. As shown in Fig. 2B, the viability of HUVECs was not affected up to $10 \mu \mathrm{M}$ of tricin treatment, indicating that the antiproliferative activity of tricin shown at range of $<10 \mu \mathrm{M}$ is not due to mere cytotoxicity of the compound.

The in vitro antiangiogenic activity of tricin. We next examined the effect of tricin on key angiogenic phenotypes such as endothelial cell invasion and tube formation. The in vitro angiogenesis assays were conducted in a non-cytotoxic concentration range of tricin $(2.5-10 \mu \mathrm{M})$. To elucidate the inhibitory activity of tricin on VEGF-induced angiogenesis, serum starved HUVECs were stimulated by VEGF with or without tricin. As shown in Fig. 3A and B, tricin significantly decreased the VEGF-induced invasiveness and tube forming ability of HUVECs in a dose-dependent manner.

Furthermore, the antiangiogenic activity of tricin was validated using a chorioallantoic membrane (CAM) assay. Coverslips containing tricin were placed on the CAM surface, and angiogenesis zones were observed under a microscope. 

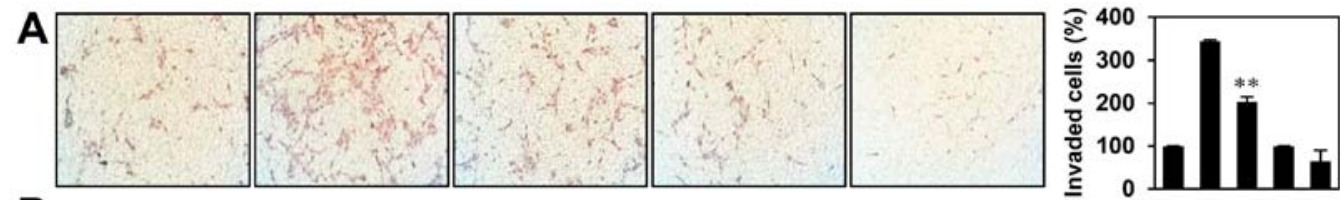

B

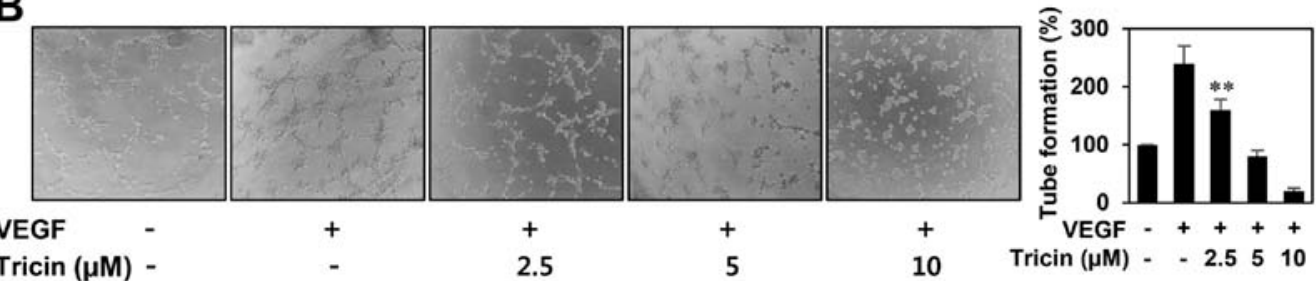

C
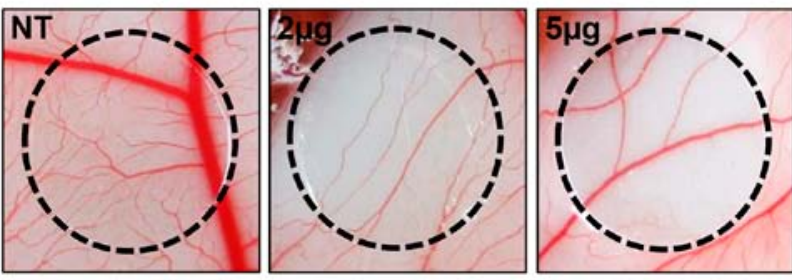

\begin{tabular}{ccc}
\hline Drug & $\begin{array}{c}\text { Inhibited egg } \\
\text { /live egg }\end{array}$ & $\begin{array}{c}\text { Inhibition } \\
\text { ratio (\%) }\end{array}$ \\
\hline NT & $2 / 15$ & 13 \\
$\begin{array}{c}\text { tricin } \\
(2 \mu \mathrm{g} / \mathrm{egg}) \\
\text { tricin }\end{array}$ & $10 / 19$ & 53 \\
$(5 \mu \mathrm{g} / \mathrm{egg})$ & $14 / 22$ & 64 \\
\hline
\end{tabular}

Figure 3. The effect of tricin on angiogenesis in vitro. (A and B) Serum-starved HUVECs were stimulated with VEGF ( $30 \mathrm{ng} / \mathrm{ml})$ in the presence or absence of tricin. (A) The inhibitory effect of tricin on the VEGF-induced invasion of HUVECs. (B) The inhibitory effect of tricin on the VEGF-induced tube-forming ability of HUVECs. The basal levels of invasiveness and tube formation of HUVECs that were incubated in serum-free medium without VEGF were normalized to $100 \%$. ${ }^{* *} \mathrm{P}<0.005$ vs. the VEGF control. (C) Antiangiogenic activity of tricin in CAMs. Fertilized chick eggs were maintained in a humidified incubator at $37^{\circ} \mathrm{C}$. At embryonic day 4.5 , coverslips loaded with vehicle alone or tricin $(2-5 \mu \mathrm{g})$ were applied to the CAM surface. Two days later, the chorioallantois was observed under a microscope. Calculations were based on the ratio of inhibited eggs relative to the total number of live eggs.

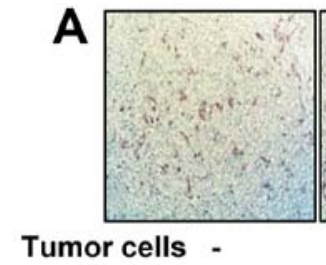

Tumor cells -
Tricin $(\mu \mathrm{M})$ -

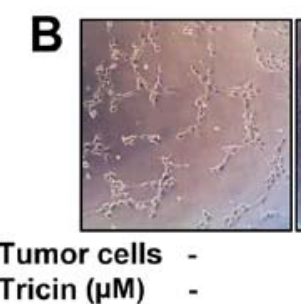

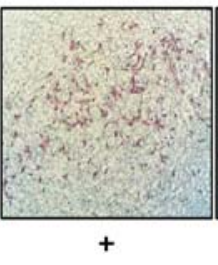

$-$

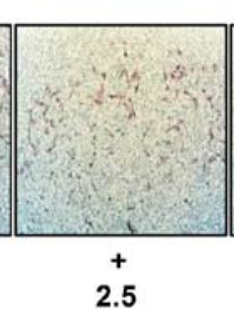

2.5

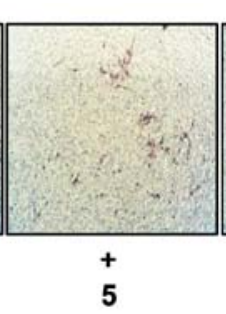

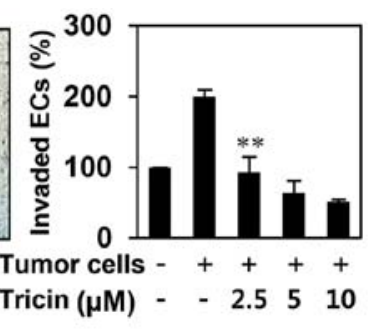

10

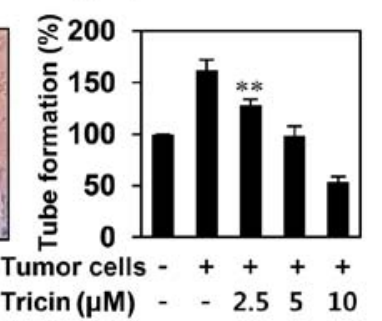

Figure 4. The effect of tricin on tumor cell-induced angiogenesis. (A and B) Tumor cell-induced angiogenesis was assessed using an in vitro co-culture system based on the chemoinvasion assay and a conditioned medium from tumor cells for in vitro tube formation assay. (A) U87MG glioblastoma cells were located at lower chamber and then were treated with tricin $(2.5-10 \mu \mathrm{M})$ for $24 \mathrm{~h}$. The medium from the lower chamber was replaced with fresh medium without tricin, and serum-starved HUVECs were placed in the upper chamber. After incubation for $18 \mathrm{~h}$, the invasiveness of HUVECs was determined by H\&E staining. The basal level of the invasiveness of HUVECs that were incubated in serum-free medium without U87MG cells was normalized to $100 \%$. ${ }^{* *} \mathrm{P}<0.005 \mathrm{vs}$. control with untreated U87MG cells. (B) U87MG cells were treated with tricin (2.5-10 $\mu \mathrm{M})$ for $24 \mathrm{~h}$, and then the medium was replaced with fresh medium without tricin. The conditioned medium was used in the in vitro tube formation assay. The basal level of the tube formation of HUVECs treated with non-conditioned medium without U87MG cells was normalized to $100 \%{ }^{* *} \mathrm{P}<0.005$ vs. conditioned medium from untreated U87MG cells.

As shown in Fig. 3C, the inhibition of neovascularization on control coverslips was $13 \%(\mathrm{n}=15)$, whereas tricin much more potently inhibited the angiogenesis of the CAM $(53 \%$ at $2 \mu \mathrm{g} / \mathrm{egg}, \mathrm{n}=19 ; 64 \%$ at $5 \mu \mathrm{g} / \mathrm{egg}, \mathrm{n}=22$ ) without toxicity against pre-existing vessels. These results demonstrate that tricin significantly inhibited angiogenesis without exhibiting cytotoxicity on endothelial cells in vitro.
The inhibitory effect of tricin on tumor cell-induced angiogenesis. Angiogenesis is recognized as a crucial step in the transition of tumors from a dormant condition to a malignant state by inducing tumor growth and metastasis $(4,5)$. To evaluate whether tricin inhibits tumor cell-induced angiogenesis, we investigated the effect of tricin on HUVEC invasion induced by U87MG glioblastoma cells using a coculture assay. 

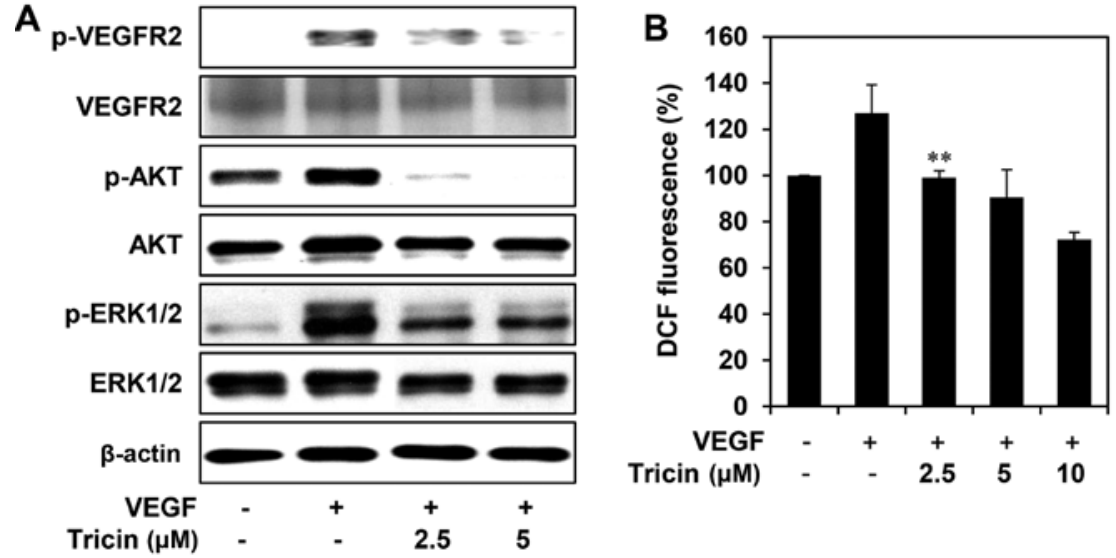

Figure 5. Downregulation of VEGFR2 signaling by tricin. (A and B) Serum-starved HUVECs were pretreated with tricin for $3 \mathrm{~h}$ at the indicated concentrations and then stimulated with VEGF (30 ng/ml) for $5 \mathrm{~min}$. (A) The effect of tricin on VEGFR2-dependent signal transduction in HUVECs. Protein levels were detected by western blot analysis. The level of $\beta$-actin was used as an internal control. (B) The effect of tricin on ROS generation in HUVECs. ROS levels were detected with $\mathrm{H}_{2}$ DCFDA. ${ }^{* *} \mathrm{P}<0.005$ vs. the VEGF control.

A

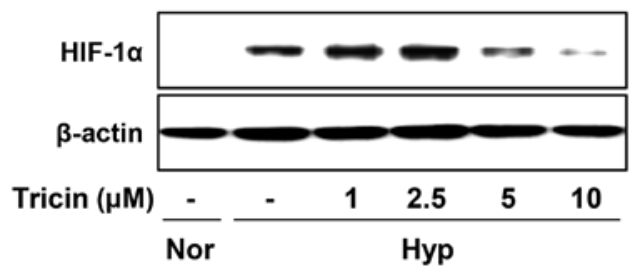

B

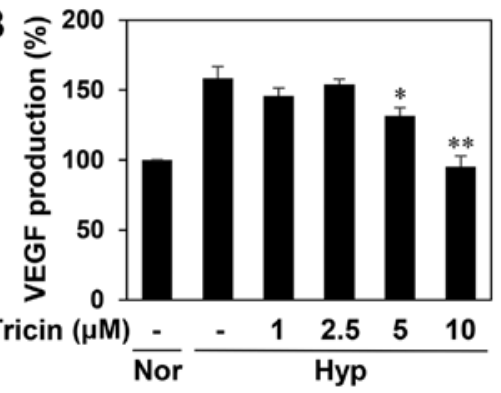

Figure 6. HIF-1 $\alpha$ inhibitory activity of tricin. (A) The effect of tricin on HIF-1 $\alpha$ protein accumulation. HepG2 cells were pretreated with tricin for $1 \mathrm{~h}$ at the indicated concentrations and then exposed to $1 \% \mathrm{O}_{2}$ for $4 \mathrm{~h}$. Protein levels were measured by western blot analysis. The level of $\beta$-actin was used as an internal control. (B) The effect of tricin on VEGF expression. HepG2 cells were pretreated with tricin for $1 \mathrm{~h}$ and then exposed to $1 \% \mathrm{O}_{2}$ for $16 \mathrm{~h}$. The concentration of VEGF protein in the culture supernatant was determined by a VEGF specific ELISA. ${ }^{*} \mathrm{P}<0.05,{ }^{* * *} \mathrm{P}<0.005$ vs. the hypoxic control.

As shown in Fig. 4A, HUVECs cocultured with U87MG cells invaded 2 -fold faster compared to HUVECs alone. The increased invasion of HUVECs was effectively prevented when U87MG cells were treated with tricin. To further verify its activity to tumor cell-induced angiogenesis, we also assessed the effect of tricin on the tube formation of HUVECs induced by U87MG cells. As shown in Fig. 4B, the conditioned medium from U87MG cells induced tube formation of HUVECs by 1.6-fold compared to control (medium only). However, tricintreated conditioned medium from U87MG cells blocked the stimulated tube formation of HUVECs in a dose-dependent manner, implying that tricin could inhibit tumor cell-induced angiogenesis.

Downregulation of VEGFR2 signal transduction by tricin. VEGFR2 signal transduction leads to the activation of various downstream signaling substrates that are involved in proliferation, migration and capillary tube formation of endothelial cells $(7,8)$. We, thus, evaluated the effect of tricin on VEGFmediated VEGFR2 signaling pathways in HUVECs. As shown in Fig. 5A, tricin efficiently suppressed the phosphorylation of VEGFR2, AKT and ERK1/2 induced by VEGF, without affecting the total protein levels, suggesting that tricin exhibits the antiangiogenic activity by inhibiting VEGFR2-mediated downstream signaling cascades. In addition, we found that tricin dose-dependently reduced the generation of ROS induced by VEGF in HUVECs (Fig. 5B). It has been previously reported that VEGF stimulates ROS production and in turn promotes VEGFR2 autophosphorylation by reversibly oxidizing and inactivating protein tyrosine phosphatases (PTPs) $(23,24)$. Taken together, these results suggest that tricin may block the VEGFR2 signaling in HUVECs via the downregulation of ROS generation.

The effect of tricin on hypoxia-induced accumulation of $H I F-1 \alpha$ protein. HIF-1 $\alpha$ pathway activation in hypoxic tumor cells is an important stimulus for tumor angiogenesis through the regulation of the expression of proangiogenic genes such as VEGF $(13,14)$. To determine the role of HIF-1 $\alpha$ in mediating the antiangiogenic effect of tricin, we evaluated the HIF-1 $\alpha$ inhibitory activity of tricin in the human hepatoma cell line HepG2, a hypervascularized tumor. As shown in Fig. 6A, tricin-treated HepG2 cells reduced the hypoxia-induced accumulation of HIF-1 $\alpha$ protein in a dose-dependent manner. We further assessed the effect of tricin on the expression of VEGF induced by hypoxia. Tricin dose-dependently decreased VEGF production in HepG2 cells under hypoxic condition (Fig. 6B). These data indicate that tricin could inhibit tumor angiogenesis by downregulating HIF-1 $\alpha$ and its target gene, VEGF. 

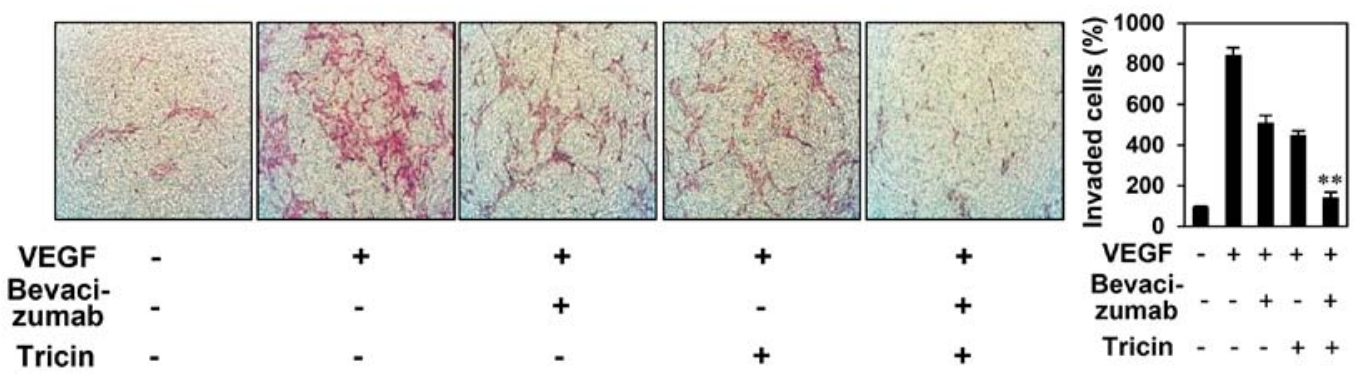

Figure 7. Enhanced antiangiogenic effect of combination treatment with bevacizumab and tricin. Serum-starved HUVECs were stimulated with VEGF $(30 \mathrm{ng} / \mathrm{ml})$ in the presence or absence of tricin $(2.5 \mu \mathrm{M})$ and bevacizumab $(100 \mathrm{ng} / \mathrm{ml})$ as indicated. The basal level of the invasiveness of HUVECs that were incubated in serum-free medium without VEGF was normalized to $100 \%$. ${ }^{* *} \mathrm{P}<0.005$ vs. the single agent treatment.

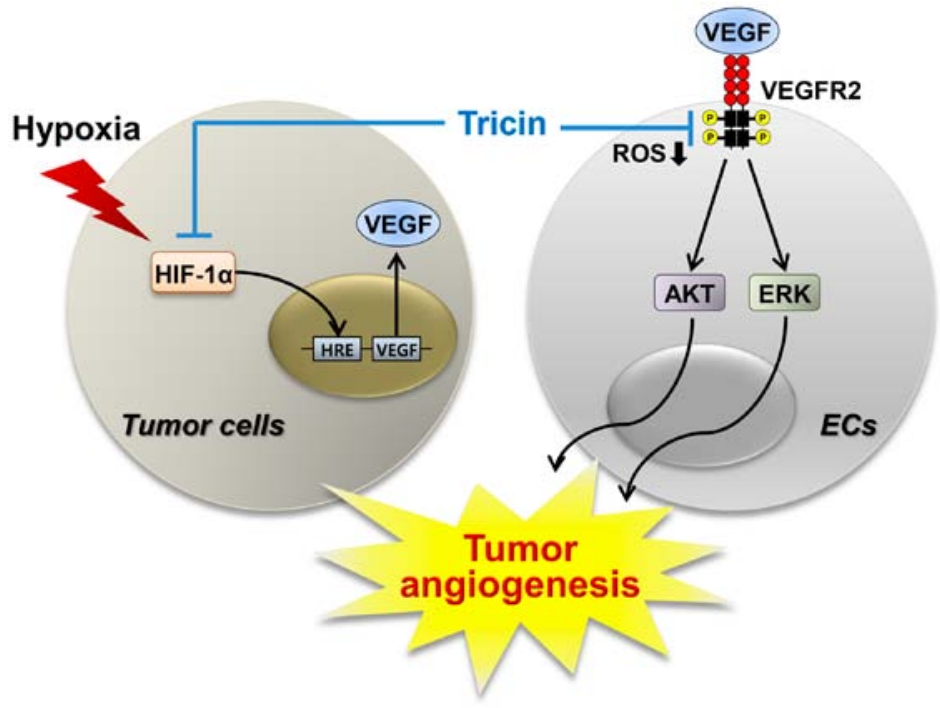

Figure 8. Role of tricin in tumor angiogenesis. The model suggests that tricin may inhibit tumor angiogenesis by modulating at least two angiogenic pathways: the blockade of VEGFR2 signal transduction in endothelial cells and suppression of the expression of HIF-1 $\alpha /$ VEGF in tumor cells.

The enhanced antiangiogenic effect of combined treatment with tricin and bevacizumab. Bevacizumab (Avastin) is a monoclonal antibody that blocks angiogenesis by inhibiting vascular endothelial growth factor (9). Although the drug has been used in treatment of various solid tumors in combination with several anticancer agents, the efficacy of this treatment is limited due to the development of resistance (25). We, thus, evaluated whether tricin elevates the antiangiogenic function of the VEGF blocker using a chemoinvasion assay. As shown in Fig. 7, treatment with both $2.5 \mu \mathrm{M}$ of tricin and $100 \mathrm{ng} / \mathrm{ml}$ of bevacizumab resulted in additive inhibition of VEGF-induced endothelial cell invasion (inhibition of 45, 53 and $94 \%$ with tricin, bevacizumab, and tricin/bevacizumab combination, respectively), suggesting that tricin potentiates the antiangiogenic activity of bevacizumab. Thus, tricin administered alone or in combination with bevacizumab may overcome the resistance to bevacizumab for antiangiogenesis.

\section{Discussion}

Owing to the crucial role of angiogenesis in the growth and metastasis of solid tumors, the specific perturbation of angiogenesis has been considered a powerful strategy for anticancer therapy. The inhibition of VEGF pathway has become the focus of antiangiogenesis research since VEGF is a pivotal stimuli of angiogenesis. Strategies to inhibit the VEGF pathway include the blockade of VEGFR2-mediated angiogenic signal transduction in endothelial cells and the prevention of VEGF expression by suppressing its transcription regulators such as HIF-1 $\alpha$ in tumor cells (26). Therefore, the novel angiogenesis inhibitors that target both VEGFR2 and HIF-1 $\alpha$ activities could provide more effective therapeutic potential for the treatment of hypervascularized tumors.

In recent years, research on dietary flavonoids has become increasingly important with the discovery of their diverse biological activities at non-toxic concentrations. Previous studies have revealed that tricin, a naturally occurring flavone, possesses antiviral, antiinflammatory, antioxidant, antitubercular, antiulcerogenic, antimelanogenic, antihistaminic and anticancer effects (27). In addition, the molecular mechanisms for its biological effects were partly identified. Tricin reduced inflammatory responses in human peripheral blood mononuclear cells (hPBMCs) by regulating the TLR4/NF- $\kappa \mathrm{B} / \mathrm{STAT}$, p38MAPK and PI3K/AKT pathways $(28,29)$. It has been also found that tricin exhibits potent anticancer effects in various cancer cells including breast and colon cancers via the inhibition of cyclooxygenase and P-glycoprotein activities $(30,31)$. The antitumor effect of tricin was also demonstrated in the 
present study. Tricin significantly inhibited the growth of human brain, gastric, colon, cervical and liver cancer cell lines (Fig. 2A). Moreover, in recent research, tricin showed pharmacokinetic benefit compared with apigenin, a known anticancer flavone. The dietary administration of tricin in mice was more available than apigenin in blood and tissues (32). Moreover, tricin did not show genotoxic properties in mice, suggesting that the safety of tricin may increase its potential clinical usefulness (33). However, to the best of our knowledge, no evaluation of the antiangiogenic activity of tricin has been reported to date.

In the present study, we describe for the first time the antiangiogenic activity and underlying molecular mechanisms of tricin. The flavone exhibited potent antiangiogenic activity in vitro with no obvious cytotoxicity (Fig. 3). We also found that tricin effectively suppressed tumor cell-induced angiogenesis (Fig. 4). Furthermore, our results demonstrated that tricin may inhibit tumor angiogenesis by modulating at least two angiogenic pathways (Fig. 8). In this study, both the VEGFR2 signaling of endothelial cells and the HIF-1 $\alpha$ and VEGF expression levels of tumor cells were downregulated by tricin treatment (Figs. 5 and 6). In addition, the blockade of VEGFR2 signal transduction may be associated with the reduction of ROS, generated from NADPH oxidase or mitochondria, by tricin.

An anti-VEGF monoclonal antibody, bevacizumab, has been approved for combination use with standard chemotherapy in certain metastatic cancers $(9,10)$. However, recent clinical results of bevacizumab have revealed its limited therapeutic efficacy in drug-resistant solid tumors as well as several adverse effects such as hypertension, proteinuria and hemorrhage $(11,12,25)$. We, thus, evaluated whether tricin ameliorates the antiangiogenic effect of bevacizumab. Combined treatment with tricin and bevacizumab more effectively inhibited VEGF-induced angiogenesis compared with single agent treatment (Fig. 7). Therefore, these results suggest that tricin may have promising therapeutic potential to overcome the resistance to bevacizumab, alone or in combination with bevacizumab.

Taken together, our results provide new therapeutic aspect for tricin as a potent inhibitor of angiogenesis via the dual blocking of VEGFR2 and HIF-1 $\alpha$ pathways. In addition, the known pharmacokinetic advantages of tricin may contribute to its clinical application to antiangiogenic therapy for cancer. Although the inhibitory effect of tricin on the VEGFR2 signaling was partially associated with the decrease of ROS generation, precise mechanisms of how tricin controls bidirectionally angiogenic pathways remain unclear. Further studies to understand the action mechanism of flavonoids will help the discovery of the upstream cellular mediators of tumor angiogenesis regulated by tricin.

\section{Acknowledgements}

We are grateful to Yonghyo Kim for his help with the CAM assay. The present study was carried out with the support of 'Cooperative Research Program for Agriculture Science and Technology Development (Project No. PJ01188001)' Rural Development Administration, Republic of Korea, the Basic Science Research Program through the National Research
Foundation of Korea (NRF) funded by the Ministry of Education (NRF-2014R1A1A2057902), and the Brain Korea 21 Plus Project, Republic of Korea.

\section{References}

1. Bussolino F, Mantovani A and Persico G: Molecular mechanisms of blood vessel formation. Trends Biochem Sci 22: 251-256, 1997.

2. Folkman J: Seminars in Medicine of the Beth Israel Hospital, Boston. Clinical applications of research on angiogenesis. NEngl J Med 333: 1757-1763, 1995.

3. Carmeliet P: Angiogenesis in health and disease. Nat Med 9: 653-660, 2003

4. Bergers $\mathrm{G}$ and Benjamin LE: Tumorigenesis and the angiogenic switch. Nat Rev Cancer 3: 401-410, 2003.

5. André T, Chastre E, Kotelevets L, Vaillant JC, Louvet C, Balosso J, Le Gall E, Prévot S and Gespach C: Tumoral angiogenesis: Physiopathology, prognostic value and therapeutic perspectives. Rev Med Interne 19: 904-913, 1998 (In French).

6. Ferrara N: VEGF and the quest for tumour angiogenesis factors. Nat Rev Cancer 2: 795-803, 2002.

7. Hicklin DJ and Ellis LM: Role of the vascular endothelial growth factor pathway in tumor growth and angiogenesis. J Clin Oncol 23: 1011-1027, 2005.

8. Holmes K, Roberts OL, Thomas AM and Cross MJ: Vascular endothelial growth factor receptor-2: Structure, function, intracellular signalling and therapeutic inhibition. Cell Signal 19: 2003-2012, 2007.

9. Ferrara N, Hillan KJ, Gerber HP and Novotny W: Discovery and development of bevacizumab, an anti-VEGF antibody for treating cancer. Nat Rev Drug Discov 3: 391-400, 2004.

10. Ivy SP, Wick JY and Kaufman BM: An overview of smallmolecule inhibitors of VEGFR signaling. Nat Rev Clin Oncol 6: 569-579, 2009.

11. Chen HX and Cleck JN: Adverse effects of anticancer agents that target the VEGF pathway. Nat Rev Clin Oncol 6: 465-477, 2009.

12. Verheul HM and Pinedo HM: Possible molecular mechanisms involved in the toxicity of angiogenesis inhibition. Nat Rev Cancer 7: 475-485, 2007.

13. Höckel M and Vaupel P: Tumor hypoxia: Definitions and current clinical, biologic, and molecular aspects. J Natl Cancer Inst 93: 266-276, 2001.

14. Forsythe JA, Jiang BH, Iyer NV, Agani F, Leung SW, Koos RD and Semenza GL: Activation of vascular endothelial growth factor gene transcription by hypoxia-inducible factor 1 . Mol Cell Biol 16: 4604-4613, 1996.

15. Semenza GL: HIF-1 and tumor progression: Pathophysiology and therapeutics. Trends Mol Med 8 (Suppl): S62-S67, 2002.

16. Semenza GL: Targeting HIF-1 for cancer therapy. Nat Rev Cancer 3: 721-732, 2003.

17. Harborne JB: Nature, distribution and function of plant flavonoids. Prog Clin Biol Res 213: 15-24, 1986.

18. Havsteen $\mathrm{BH}$ : The biochemistry and medical significance of the flavonoids. Pharmacol Ther 96: 67-202, 2002.

19. Cai H, Hudson EA, Mann P, Verschoyle RD, Greaves P, Manson MM, Steward WP and Gescher AJ: Growth-inhibitory and cell cycle-arresting properties of the rice bran constituent tricin in human-derived breast cancer cells in vitro and in nude mice in vivo. Br J Cancer 91: 1364-1371, 2004.

20. Hudson EA, Dinh PA, Kokubun T, Simmonds MS and Gescher A: Characterization of potentially chemopreventive phenols in extracts of brown rice that inhibit the growth of human breast and colon cancer cells. Cancer Epidemiol Biomarkers Prev 9: $1163-1170,2000$

21. Oyama T, Yasui Y, Sugie S, Koketsu M, Watanabe K and Tanaka T: Dietary tricin suppresses inflammation-related colon carcinogenesis in male Crj: CD-1 mice. Cancer Prev Res (Phila) 2: 1031-1038, 2009.

22. Ali MA, Choy H, Habib AA and Saha D: SNS-032 prevents tumor cell-induced angiogenesis by inhibiting vascular endothelial growth factor. Neoplasia 9: 370-381, 2007.

23. Ushio-Fukai M: VEGF signaling through NADPH oxidasederived ROS. Antioxid Redox Signal 9: 731-739, 2007.

24. Jung HJ, Kim Y, Chang J, Kang SW, Kim JH and Kwon HJ: Mitochondrial UQCRB regulates VEGFR2 signaling in endothelial cells. J Mol Med (Berl) 91: 1117-1128, 2013.

25. Bergers G and Hanahan D: Modes of resistance to anti-angiogenic therapy. Nat Rev Cancer 8: 592-603, 2008. 
26. El-Kenawi AE and El-Remessy AB: Angiogenesis inhibitors in cancer therapy: Mechanistic perspective on classification and treatment rationales. Br J Pharmacol 170: 712-729, 2013.

27. Zhou J and Ibrahim RK: Tricin - a potential multifunctional nutraceutical. Phytochem Rev 9: 413-424, 2010.

28. Shalini V, Pushpan CK, Sindhu G, Jayalekshmi A and Helen A: Tricin, flavonoid from Njavara reduces inflammatory responses in hPBMCs by modulating the p38MAPK and PI3K/Akt pathways and prevents inflammation associated endothelial dysfunction in HUVECs. Immunobiology 221: 137-144, 2016.

29. Shalini V, Jayalekshmi A and Helen A: Mechanism of antiinflammatory effect of tricin, a flavonoid isolated from Njavara rice bran in LPS induced hPBMCs and carrageenan induced rats. Mol Immunol 66: 229-239, 2015.

30. Cai H, Al-Fayez M, Tunstall RG, Platton S, Greaves P, Steward WP and Gescher AJ: The rice bran constituent tricin potently inhibits cyclooxygenase enzymes and interferes with intestinal carcinogenesis in ApcMin mice. Mol Cancer Ther 4 1287-1292, 2005.
31. Jeong YH, Chung SY, Han AR, Sung MK, Jang DS, Lee J, Kwon Y, Lee HJ and Seo EK: P-glycoprotein inhibitory activity of two phenolic compounds, (-)-syringaresinol and tricin from Sasa borealis. Chem Biodivers 4: 12-16, 2007.

32. Cai H, Boocock DJ, Steward WP and Gescher AJ: Tissue distribution in mice and metabolism in murine and human liver of apigenin and tricin, flavones with putative cancer chemopreventive properties. Cancer Chemother Pharmacol 60: 257-266, 2007.

33. Verschoyle RD, Greaves P, Cai H, Borkhardt A, Broggini M, D'Incalci M, Riccio E, Doppalapudi R, Kapetanovic IM, Steward WP, et al: Preliminary safety evaluation of the putative cancer chemopreventive agent tricin, a naturally occurring flavone. Cancer Chemother Pharmacol 57: 1-6, 2006. 